\title{
Review Paper \\ The Effectiveness of Preoperative Exercises on the Outcomes After Anterior Cruciate Ligament Reconstruction: A Systematic Review
}

\author{
Ali Yalfani ${ }^{1}\left(\mathbb{C}\right.$, Ahmad Ebrahimi Atri $^{2} \mathbb{C}^{\circ},{ }^{*}$ Maedeh Taghizadeh Kermani ${ }^{1}$ (i)
}

1. Department of Corrective Exercise and Sport Injury, Faculty of Physical Education and Sport Sciences, Bu Ali Sina University, Hamedan, Iran. 2. Department of Sport Physiology, Faculty of Sport Sciences, Ferdowsi University, Mashhad, Iran.

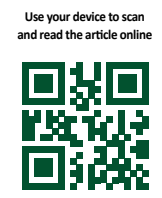

Citertion: Yalfani A, Ebrahimi Atri A, Taghizadeh Kermani M. [The Effectiveness of Preoperative Exercises on the Outcomes After Anterior Cruciate Ligament Reconstruction: A Systematic Review (Persian)]. Journal of Sport Biomechanics. 2019; 5(3):188-198. https://doi.org/10.32598/biomechanics.5.3.6

doi) $h$ https://doi.org/10.32598/biomechanics.5.3.6

Key words:

Preoperative exercises, Anterior cruciate ligament injury, Knee extensor strength, Knee function

\section{ABSTRACT}

Objective Quadriceps weakness is common after Anterior Cruciate Ligament (ACL) injury and subsequent surgery. Preoperative defects affect postoperative outcomes. The purpose of this review study was to investigate whether preoperative exercises can affect the postoperative outcomes after $\mathrm{ACL}$ reconstruction.

Methods The searching for papers was conducted in the PubMed database among the studies from 1990 to 2019 using following keywords: ACL preoperative exercise, prehabilitation ACL, and preoperative protocol. The initial search yielded 442 papers. Then, based on the inclusion and exclusion criteria, 10 papers were selected for the final review. Methodological quality of the studies was assessed by using the Physiotherapy Evidence Database (PEDro) scale and the Critical Appraisal Skills Program (CASP).

Results Some preoperative exercises (traditional, strength, and neuromuscular) can significantly increase the knee extensor strength and knee function in men and women after surgery in the short and long periods. The mean PEDro score for 8 randomized controlled trials was 6 , indicating their moderate methodological quality. Moreover, the CASP score for 2 cohort studies was 10 and 11 out of 12 .

Conclusion Preoperative rehabilitation consisting of neuromuscular and strength exercises followed by a criterion-based postoperative rehabilitation program cause greater functional outcomes and faster recovery of the knee extensor strength after $A C L$ reconstruction. Preoperative rehabilitation should be considered as a standard treatment to maximize functional outcomes after $\mathrm{ACL}$ reconstruction.

\section{Extended Abstract}

\section{Introduction}

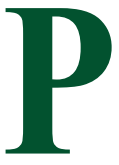

ersistent quadriceps weakness has been reported as one of the limiting factors in returning to pre-injury competition level, which can last for more than 2 years after surgery. Weakness of the quadriceps not only causes abnormal gait and loss of function, but may also be a factor in knee osteoarthritis. There is a weakness in the quadriceps in reducing the angle and torque of the knee in walking following Anterior Cruciate Ligament (ACL) reconstruction. The preoperative phase may be helpful in reducing the risk of postoperative complications and improving successful return to high-level activity. To our knowledge, this is the first systematic review study conducted to evaluate the ef-

\section{* Corresponding Author:}

Maedeh Taghizadeh Kermani, PhD.

Address: Department of Corrective Exercise and Sport Injury, Faculty of Physical Education and Sport Sciences, Bu Ali Sina University, Hamedan, Iran. Tel: +98 (915) 4486601

E-mail: maedeh.taghizadeh1988@gmail.com 
fectiveness of preoperative rehabilitation protocols to improve patient outcomes after ACL reconstruction.

\section{Methods}

This systematic review was conducted using PRISMA (Preferred Reporting Items for Systematic Reviews and Meta-Analyses) statement guidelines. The searching was conducted in PubMed database using following keywords: ACL Preoperative exercise, Prehabilitation ACL, preoperative Protocol. The initial search yielded 442 articles on ACL reconstruction. The papers were then examined based on the entry criteria. After checking the titles, abstracts and full texts of the articles, 10 articles on the effect of preoperative ACL exercises on the postoperative outcomes were identified. Entry criteria were related to studies that examined the effects of preoperative ACL exercises on postoperative results whose designs were randomized clinical trial, observational cohort, and case-control. Studies that had no pre-surgery training protocol (with only post-surgery exercises), case reports, and review articles were omitted. Table 1 shows the criteria for entering the study based on PICO method. Physiotherapy Evidence Database (PEDro) scale was used for randomized studies to evaluate the quality of their methodology. For cohort studies, the Critical Appraisal Skills Program (CASP) was applied.

\section{Results}

The PEDro score of randomized studies ranged from 3 to 7 out of 10 (mean score $=6$ ), indicating a moderate methodological quality; there were 2 studies with low quality, 4 with moderate quality and 2 with high quality. The CASP score of the two cohort studies was 10 and 11 out of 12 . Most studies had evaluated the effect of a preoperative ACL exercises program on the isokinetic strength of the knee. One study showed a significant difference in quadriceps strength between patients received preoperative ACL exercises (range of motion, strength and balance exercises) and those without preoperative exercises after surgery. In a study, a significant difference in maximum torque and quadriceps muscle function was observed in the intervention group that performed isokinetic strength training at 60120 degrees per second for 4 weeks before surgery [25].

Logerstedt et al. examined the relationship between quadriceps strength before surgery and knee function after surgery. They found that quadriceps strength before surgery was a significant indicator of the International Knee Documentation Committee 2000 (IKDC 2000) score 6 months after surgery, and quadriceps weakness before surgery can affect the knee function after 6 months. Knee function was assessed using single-leg jump and a functional questionnaire. Three studies showed a significant difference between intervention and control groups. Kim et al. and Shaarani showed a significant increase in performance (during single-leg jump and using a functional questionnaire) in the intervention group who received preoperative exercise (range of motion, balance, and strength) compared to the controls. Filla et al. showed that in DOC University patients, there was a significant improvement in the IKDC 2000 and Knee Osteoarthritis outcome Score 2 years after surgery.

\section{Discussion}

The review of the studies showed that the groups that had received a preoperative exercise program showed a significant improvement in knee function and physical performance after surgery compared to the controls. It has been suggested that performing preoperative exercises (strength + neuromuscular) and achieving a normal range of motion, activating the quadriceps, reducing pain and swelling can lead to significant progress after two years of ACL reconstruction. Weakness in knee extensor strength after surgery is a common problem that has been reported in previous studies. The rapid decline in the function of the quadriceps, which occurs mainly in the early postoperative period, is caused by arthrogenic muscle inhibition due to pain, inflammation, swelling,

Table 1. The inclusion criteria based on PICO method

\begin{tabular}{cc}
\hline Pico Indicators & Inclusion Criteria \\
\hline Design & Randomized controlled trial and cohort \\
\hline Population & Participants with acute unilateral ACL injury (male and female) with no age restrictions \\
\hline Intervention & Preoperative ACL exercises (neuromuscular, strength, balance and range of motion) \\
\hline Comparisons & With no preoperative exercises and traditional exercises \\
\hline Outcome measures & Knee strength, knee function and quality of life \\
\hline & \\
\hline
\end{tabular}


as well as impairment in the proprioceptors of the joint. This neurological mechanism for weakness of the quadriceps may persist for a long time after injury or ACL surgery.

\section{Conclusion}

Rehabilitation exercises before ACL reconstruction (neuromuscular and strength exercises) can increase knee function based on a single-leg jump test and functional questionnaire scores. It can increase the quadriceps strength in the short and long periods after surgery. Maximizing the strength of the quadriceps with advanced preoperative exercises should be a major goal for specialists to improve knee function after ACL surgery.

\section{Ethical Considerations}

Compliance with ethical guidelines

This study is a systematic review and there is no need for ethical approve.

Funding

This research did not receive any specific grant from funding agencies in the public, commercial, or notfor-profit sectors.

Authors' contributions

Investigation, resources, draft preparation: Maedeh Taghizadeh Kermani; Review and edit: Maedeh Taghizadeh Kermani, Ali Yalfani; Study appraisal: Maedeh Taghizadeh Kermani, Ahmad Ebrahimi Atri.

Conflicts of interest

The authors declare no conflict of interest. 


\title{
اثربخشى تمرينات قبل از جراحى بر نتايج يس از بازسازى رباط صليبى قدامى:مرورى سيستماتيك
}

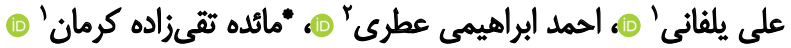 \\ 1. كروه آسيبشناسى ورزشى و حركات اصلاحي، دانشكده تربيثبدنى و علوم ورزشى، دانشكاه بوعلى سينا، همدان، ايران.

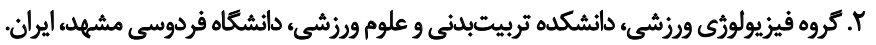

\begin{abstract}
حكيد

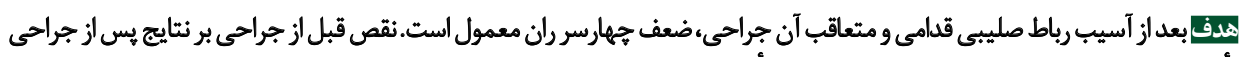

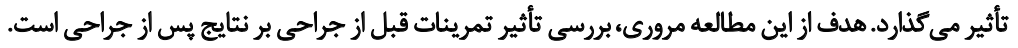

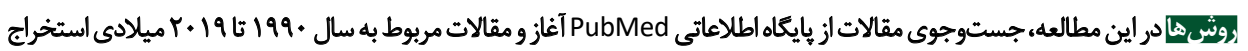

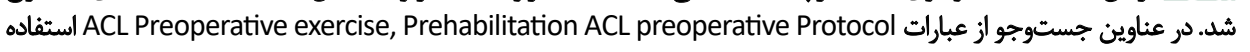

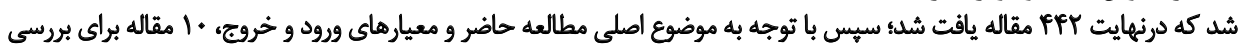

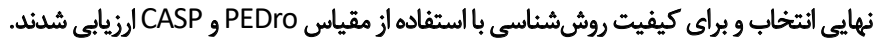

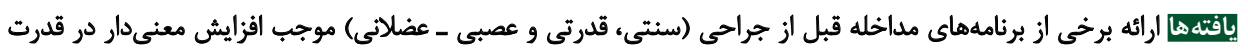

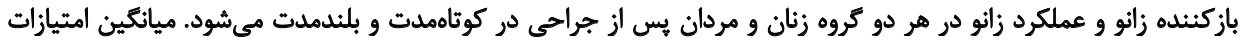

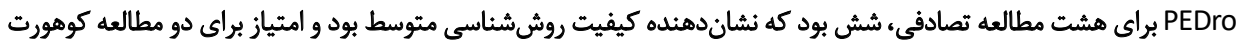

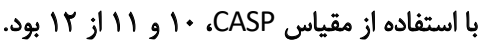

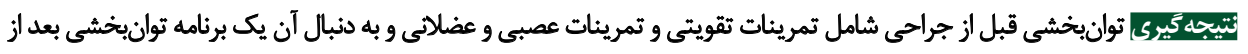

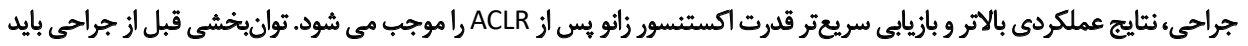

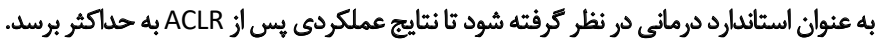

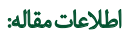

تاريخ دريافت: Trav

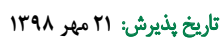

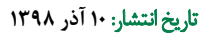

كإن تمريثات قبل از جراحي، آسيب رباط صليبي قدي إزي

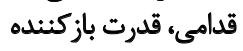

زانو، عملكرث زائو

را نشان داده است، نقص ماندكار در قدرت جهمارسر ران به عنوان

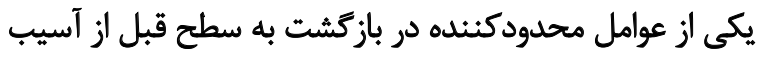

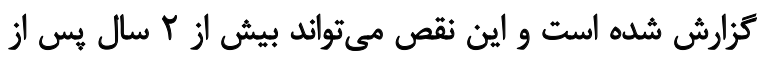

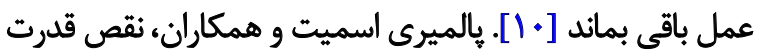

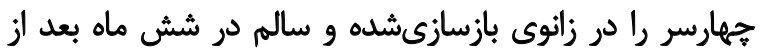

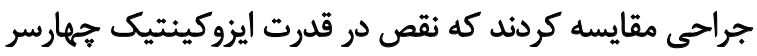

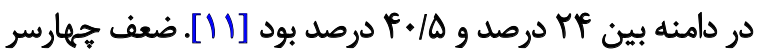

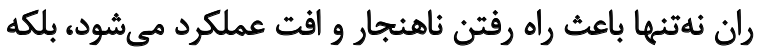

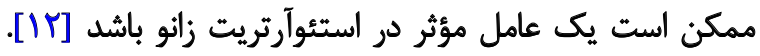

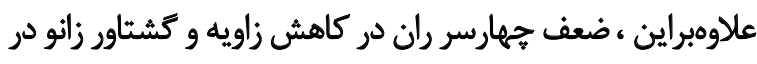

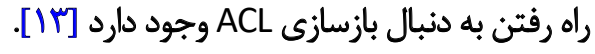

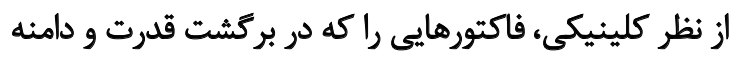

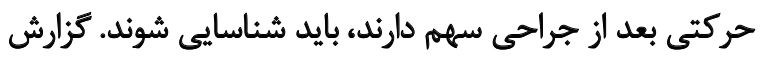

dateo

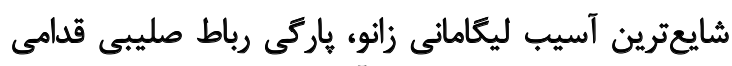

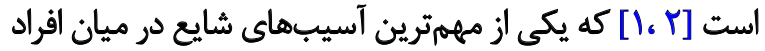

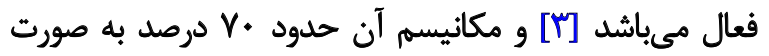

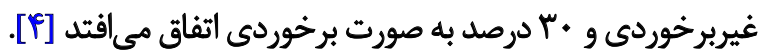

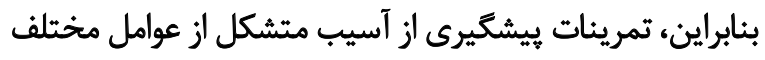

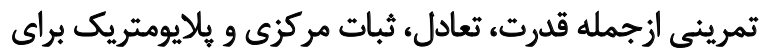

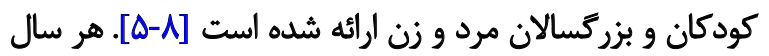

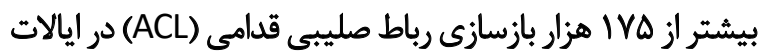

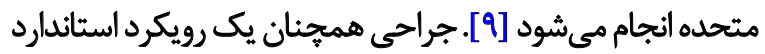

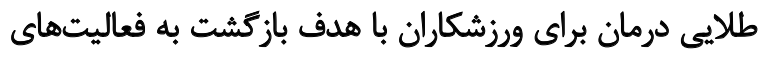

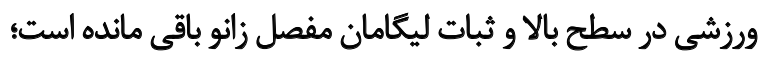

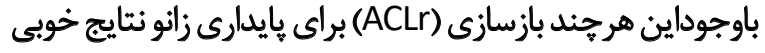

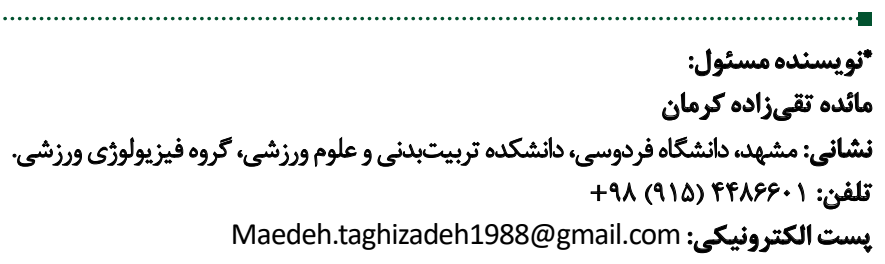




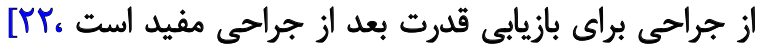

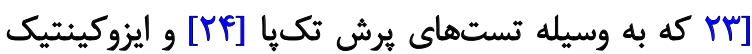

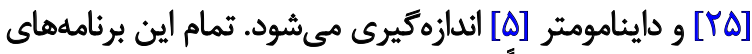

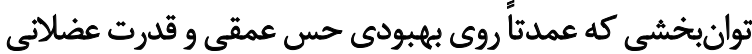

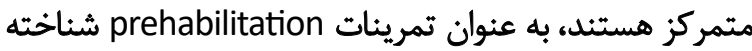

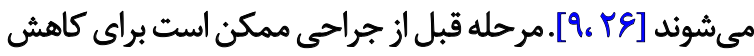

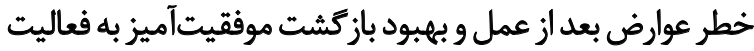

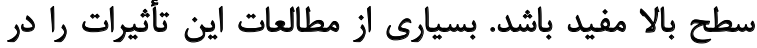

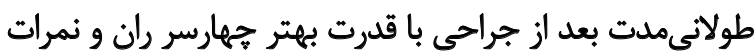

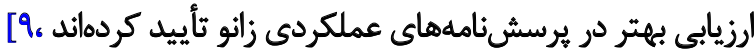

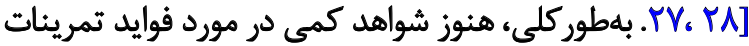

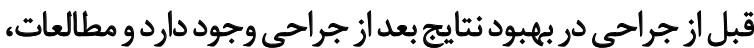

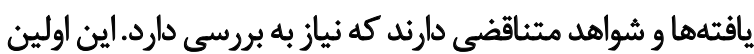

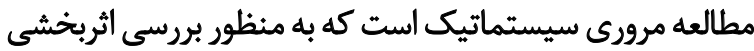

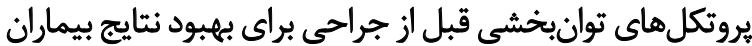
بعد از بازسازى انجام شده است.

$$
\text { روش استراتثى جستوجى }
$$

PRIS- اين بررسى سيستماتيك با استفاده از دستورالعمل إنهاى

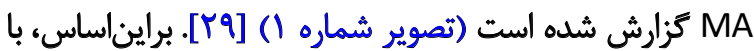

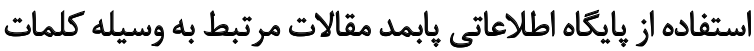

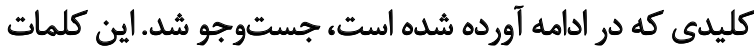
عبارت بودند از: - ACL Preoperative exercise, Pre habilita tion ACL, preoperative Protocol بعد از بررسى اوليه، فهرست همه مقالات بر اساس معيارهاى
شده است كه به تأخير انداختن جراحى به مدت سه تا جاتهار

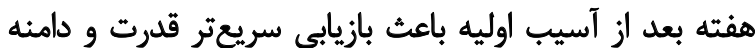

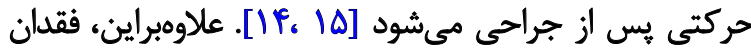

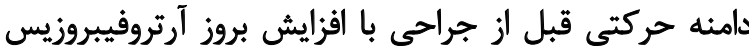

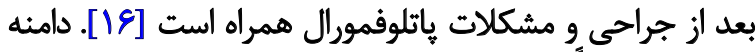

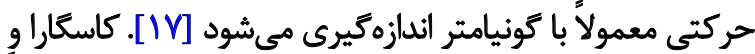

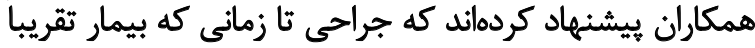

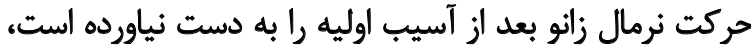

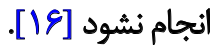
بازكشت به ورزش به عوامل مختلفى ازجمله قدرت، دامنه

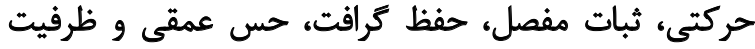

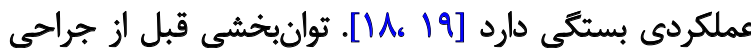

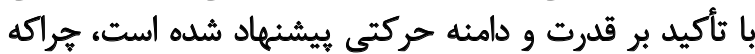

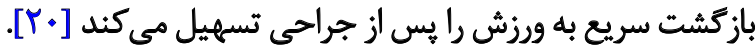

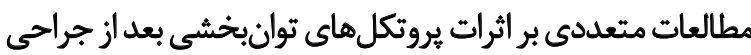

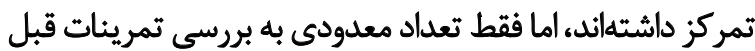

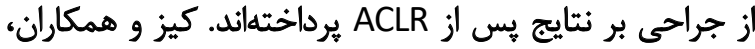

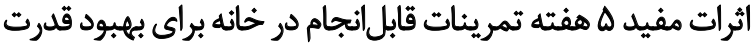

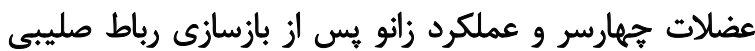

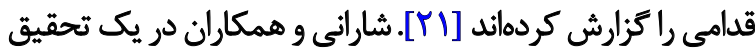

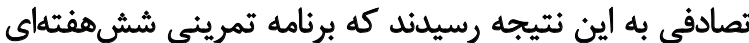

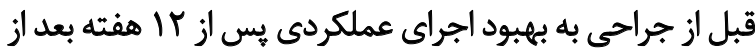

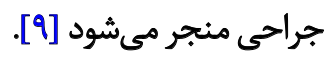

كزارش شده است كه افزايش قدرت جهارسر قبل از جراحي

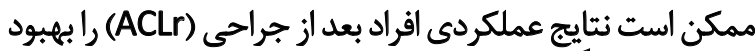

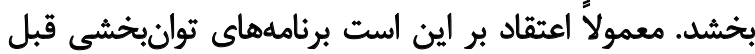

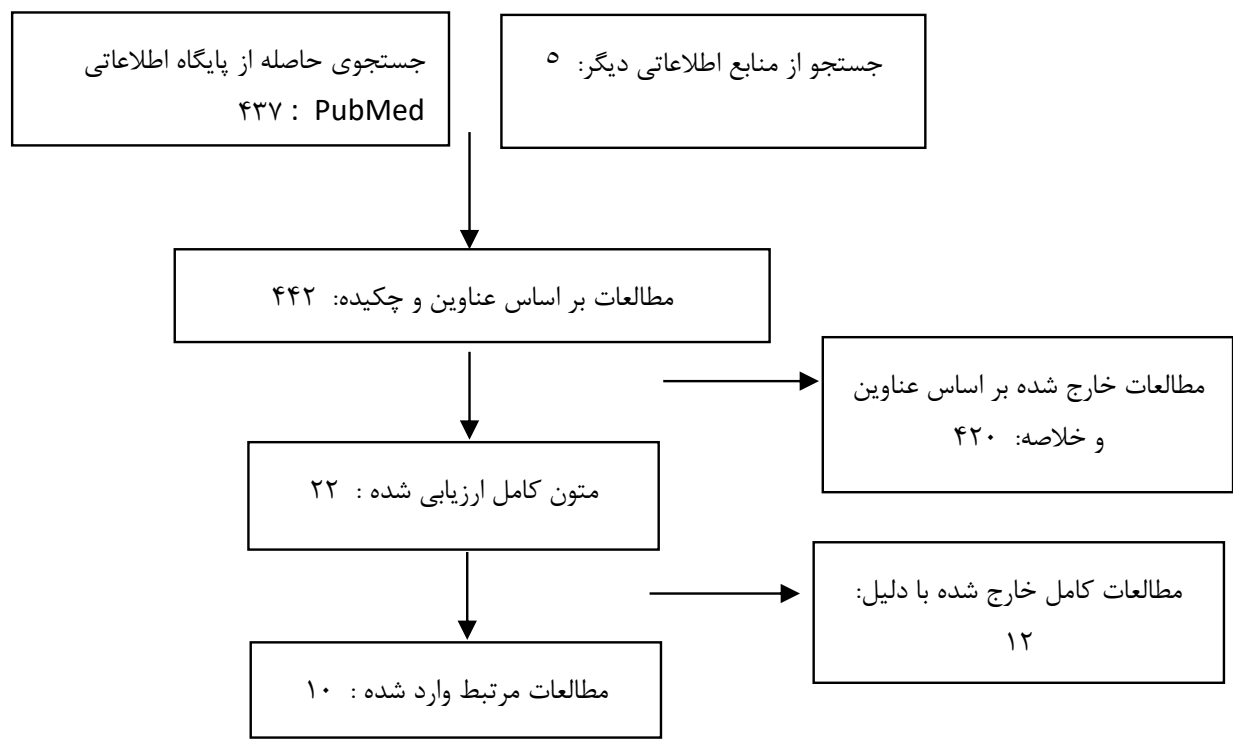


از Critical Appraisal Skills Program² انجام شد [1/r]. امتياز

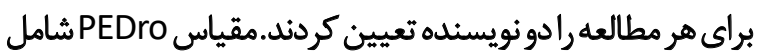

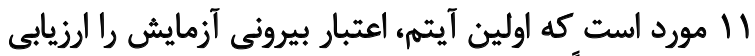

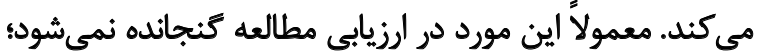

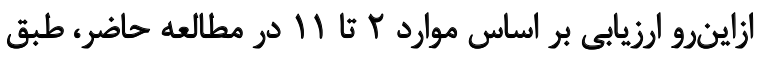

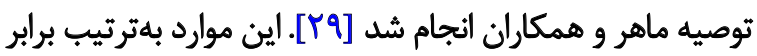

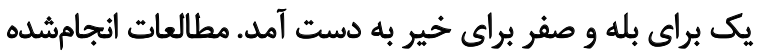

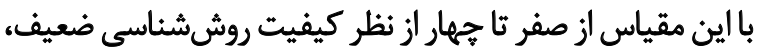

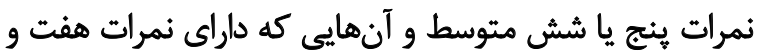

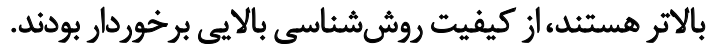

نثايج

درمجموع، · 1 مطالعه كه در ينج كشور مختلف انجام شده

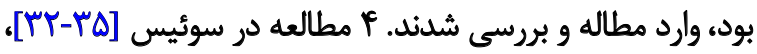

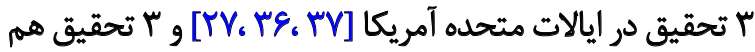

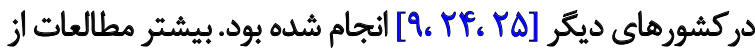

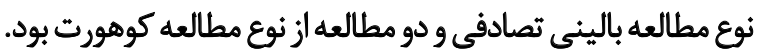

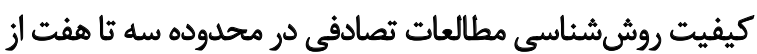

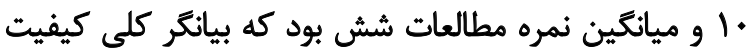

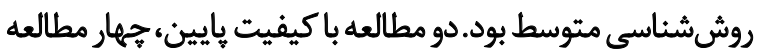

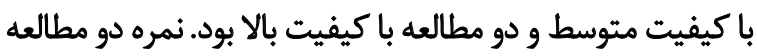

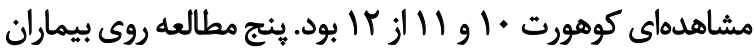

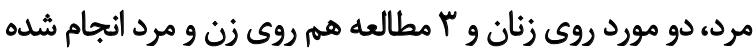

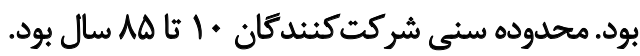

هروتكل تمرينات قبل از جراحى در مدت، تكرار و محتوا با

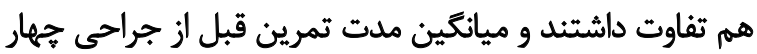

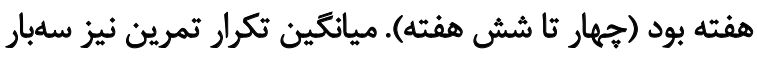

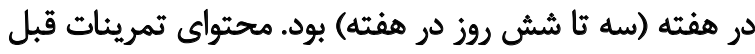

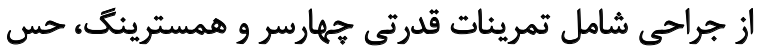

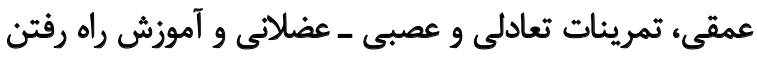

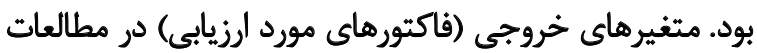

2. CASP
ورود و خروج به منظور يبيدا كردن اطلاعات اضافى بررسى شد.

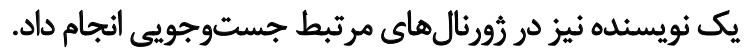

(e.g., The American Journal of Sports Medicine, Journal of Orthopaedic \& Sports Physical Therapy, Medicine and Science in Sports and Exercise, British Journal of Sports Medicine, Journal physical therapy science)

استراترى كامل جستوجو در تصوير شماره ا مشاهده مى رشود.

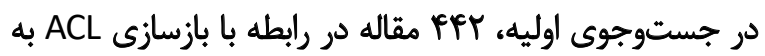

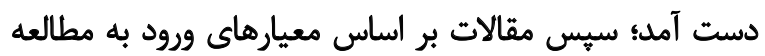

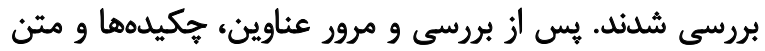

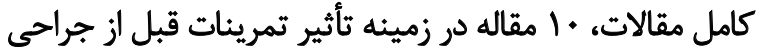

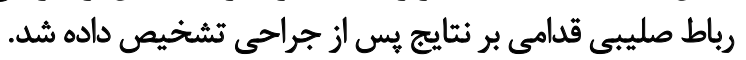

\section{معيار ورودو وخروج}

دو مروركر به طور مستقل، خكيده و عناوين را با توجه به

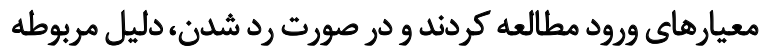

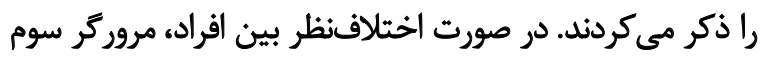

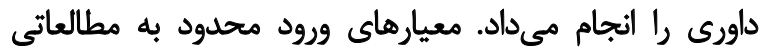

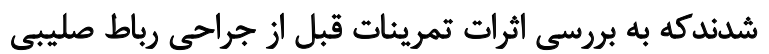

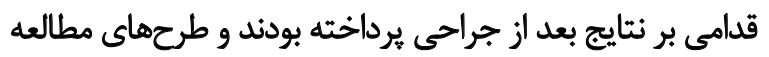

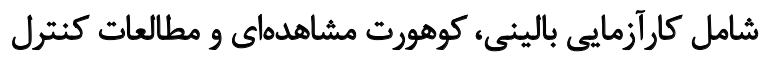

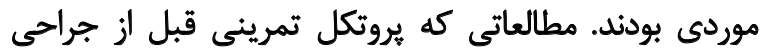

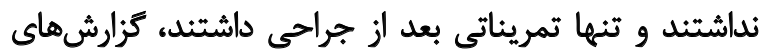

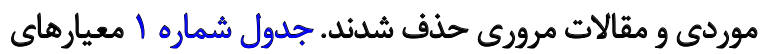

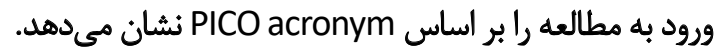

$$
\text { ارزيابى كيفى مطالعه }
$$

Physiothera- براى ارزيابى كيفيت روششناسى از مقياس py Evidence Database ${ }^{1}$ [بّ]. از طرف ديكر، ارزيابى روش مطالعات كوهورت با استفاده

1.PEDro

جدول ا. معيار ورود بر طبق PICO acronym

\begin{tabular}{|c|c|}
\hline شاخمص ييكو & Results According PICO \\
\hline 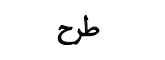 & مطالعات تصادفى و كوهورت \\
\hline جامعل & شركتكنندكان با آسيب حاد يكطرفه ACL (زنان و مردان) بلون محلوديت سنى \\
\hline مداخله & تمرينات قبل از جراحى (عصبى ـ عضلانى، قدرتى، تعادلى و دامئه حركتى) \\
\hline مقايسه & بلون تمرين قبل از جراحى و تمرينات ستىى \\
\hline ت نتّائج & قلرت زاثو، عملكرد زأو و كيفيت زندكى \\
\hline
\end{tabular}

مجله بيومكانيك وزنش 
جدول r. خلاصه مطالعات صورت كرفته

\begin{tabular}{|c|c|c|c|c|c|}
\hline ثتايج & ارزيابي إيبكيرى & مداخله & مثغير اندازهيري شُهد & نمونها & هنيع \\
\hline 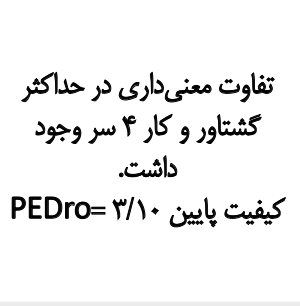 & تمرينات قبل ازئ & 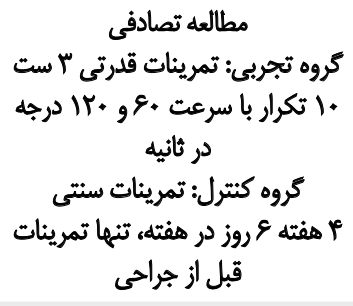 & 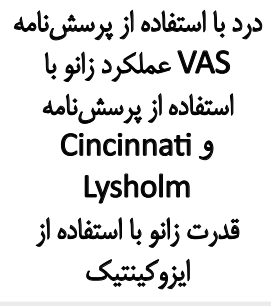 & 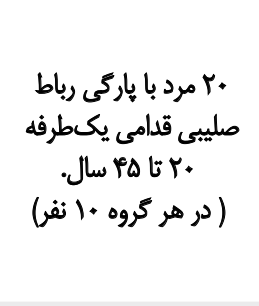 & {$[r \Delta]$} \\
\hline 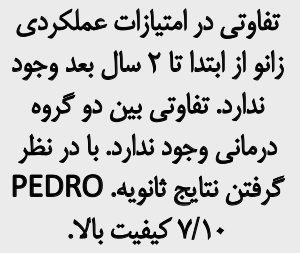 & 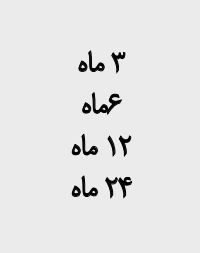 & 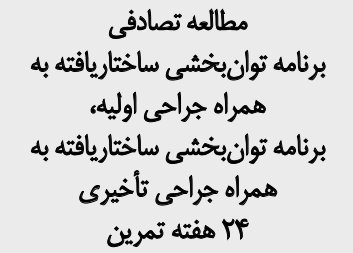 & 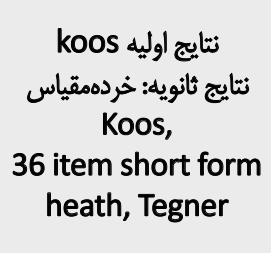 & 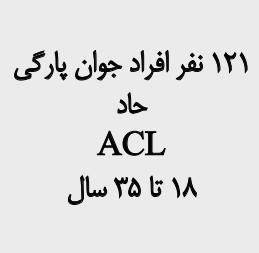 & {$[\mathrm{H}]$} \\
\hline
\end{tabular}

نقص در قدرت زأو در •و مور

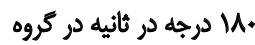
تجربى در مقايسه با كروه درو كتثرل در با ماه بعد از جراحي به طور معنى دارى بايينتر

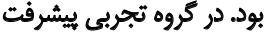
تمعنى دار در تست مسافت بيرش تكيا وجود داثشت (sEDro) .(score

.

\section{حلاكثر كشتاور جهارسر در اندام آسيبديده در مقايسه با ابتدا بهبود يييدا كرد. اكرجه در مقايسه با كروه كنترل امتيازات عملكردى زانو معنى دار نبود \\ قبل از جراحى

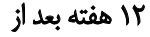 جراحى$$
\text { نبود }
$$

\section{مجموعه تستهاي

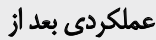 تمرينات قبل ازئ تمبردي جراحي، يرسش تامئه F أ ماه بعد ازئ IKDC \\ جر:}

\section{ثاخص قلرت زانو در عاه} ماه بعد از جراحى در هر درد دون كروه بهبود بييدا كرد كرو درو Son cooper |غتشتشاشي دريافت كرده بودند. excursion بين أندامهايشان در \& ماه بعد از جراحى تفاوتى بنان نداشت.

SEDro كيفيت بايين 1./\% :score

\section{مطالعه تصادفى

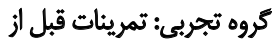

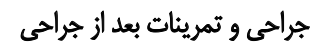

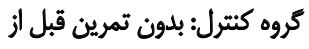
جراحى و تمرينات بعد از جراحى

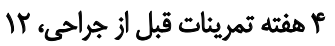
هفته تمرينات بعل أز جراحيى

$$
\begin{aligned}
& \text { قدرث و عملكرد زانو } \\
& \text { A بيمار مرد با ميائكين } \\
& \text { سنى Tr سال } \\
& \text { در هر كروه •. أنقر سال }
\end{aligned}
$$

مطالعه طولى مبري برئامه تمرينى قبل از جراحى تمريثات قدرتى (شيدت بالا و تكرار كم) + تمرينات اغتشاشي و و والات وتكرار تمرينات بعد از جراحى +1 جلسئ تمرينات ثبل از جراحى و و ماهي تهاه تمرينات بعد از جراحى اجى

$$
\text { تصادفي }
$$

كروه تجربي: تمرينات قبل ازئ جراحى قابل انجام در خانه و باثشكاه كروه كتثرل: بلدون تمرينات قبل از جراحى بلدي

هر دو كروه تمرينات بعد از جراحيى

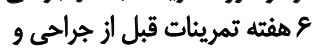
Ir هفته تمرينات بعد از جراحي جئ

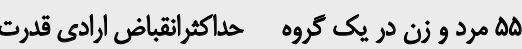

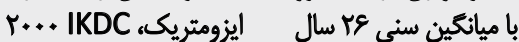

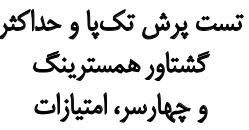
يرسش نامه عملكردى زانو ا نفر در هر كروها
$[\mathrm{TV}]$

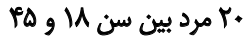

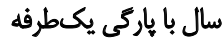
ACL

$$
\begin{aligned}
& \text { تصادفى } \\
& \text { تمرينات قدرتى با شدت بالا و تكرار } \\
& \text { بعد از +1 جلسه } \\
& \text { تمرينات قبل ازل جله } \\
& \text { جراحيى } \\
& \text { ع ماه بعد از جراحى }
\end{aligned}
$$

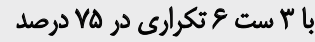

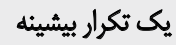

$$
\begin{aligned}
& \text { تمرينات قدرتي+|غتشاشي يكي } \\
& \text {. جلسه تمرينات قبل از جراحى و } \\
& \text { عاه تمرين }
\end{aligned}
$$

$$
\begin{aligned}
& \text { non } 2 \text { و بر } 19 \\
& \text { cooper } \\
& \text { ه IV ACL } \\
& \text { جنفر تمرينات اغتشاشي }
\end{aligned}
$$

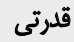

$$
\begin{aligned}
& \text { ا نقفر تمرينات قلرتى }
\end{aligned}
$$

شاخص قدرت زانو،

Excursion knee

فاز مياني راه رفتن 


\begin{tabular}{|c|c|c|c|c|c|}
\hline ثتايج & ارزيابى / ييّيّيرى & مداخله & متغير اتندازهيري شُشه & نمونهها & منيع \\
\hline 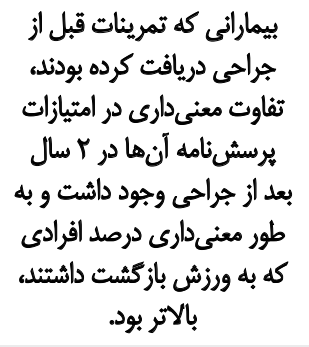 & إبثال & 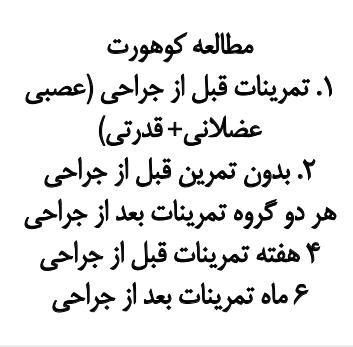 & $\begin{array}{c}\text { يرسش نامdهاي (IKDC) } \\
\text { (KOOS) }\end{array}$ & 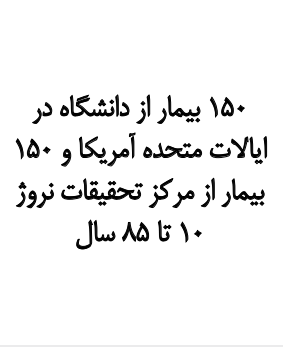 & [rq] \\
\hline 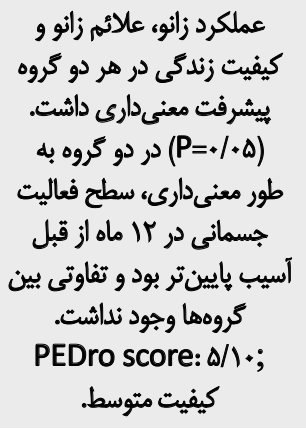 & 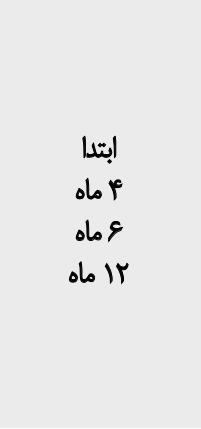 & 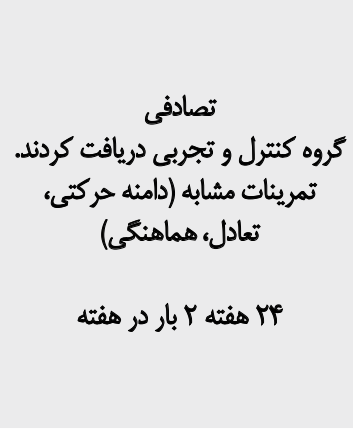 & 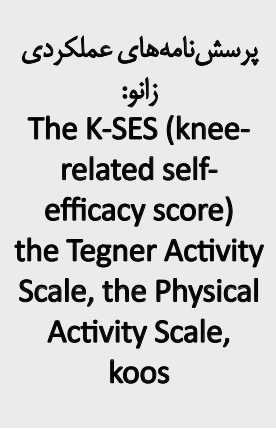 & 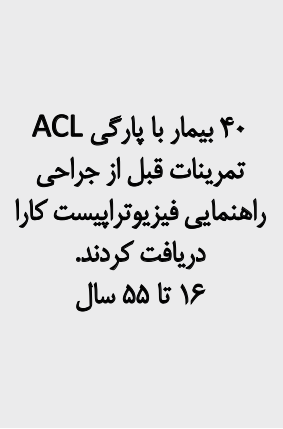 & [Ta] \\
\hline 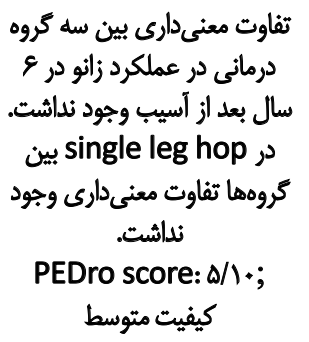 & 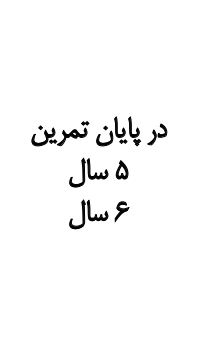 & 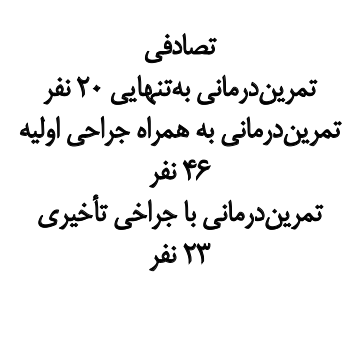 & 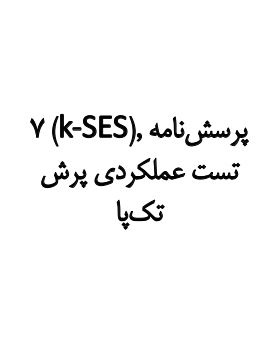 & 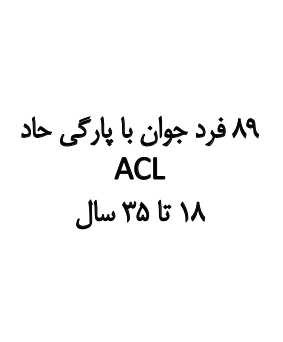 & {$\left[\mathrm{Mr}^{c}\right]$} \\
\hline 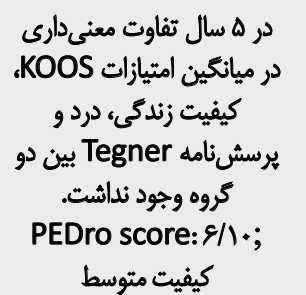 & هـ سال & 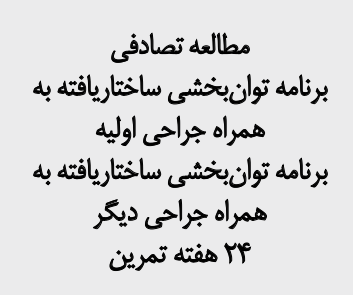 & 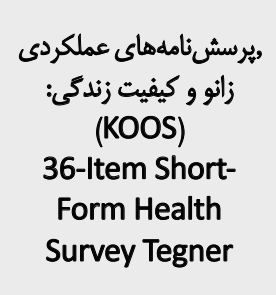 & 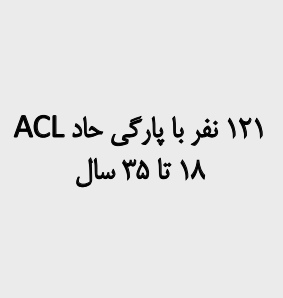 & {$[\mathrm{HT}]$} \\
\hline
\end{tabular}

مجله بيومكانيك ورنث

جراحى و عملكرد زانو بعد از جراحى را بررسى كردند و به اين جاين

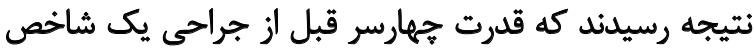

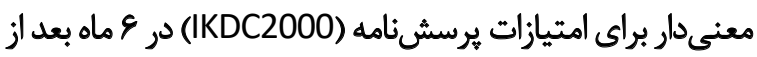

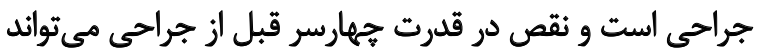

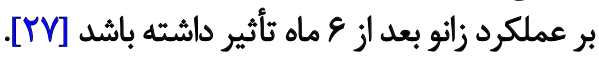

در مطالعه هرتيگان شاخص قدرت زانو با استفاده از حداكثر

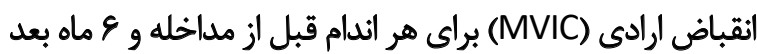

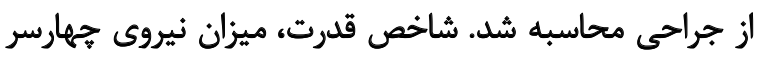

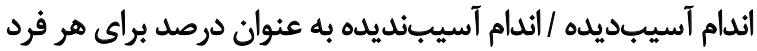

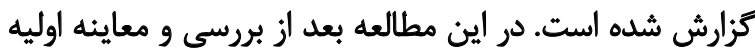

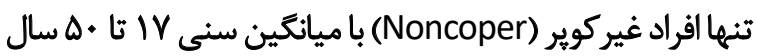

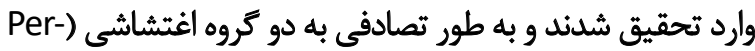

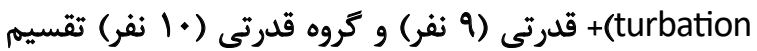

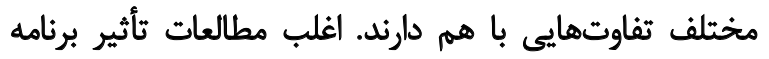

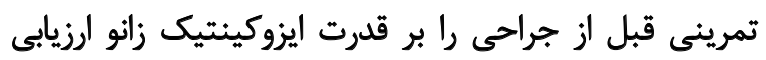

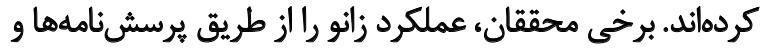

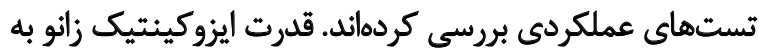
عنوان يك خروجى در ه مقاله اندازمكيرى شدرد يك مطالعه تفاوت معنى دار بين بيماران در تروه تمرينات قبل تريل

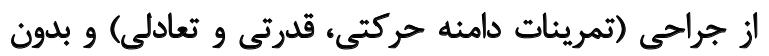

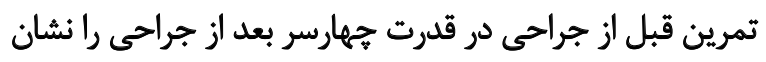

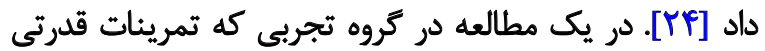

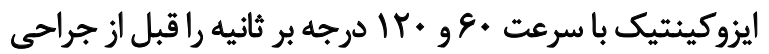

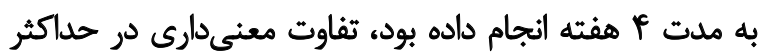

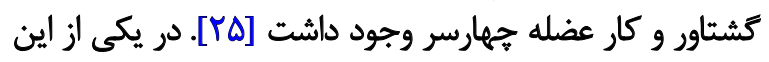

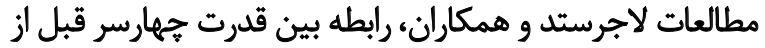


جراحى يك مشكل معمول است كه در مطالعات قبلى كَزارش

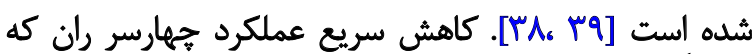

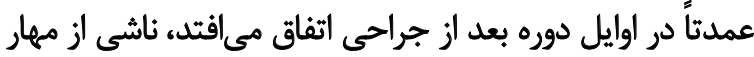

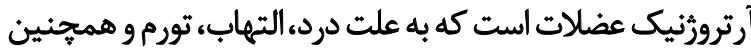

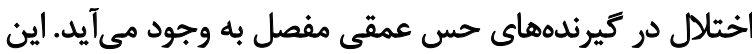

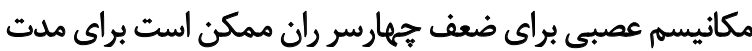

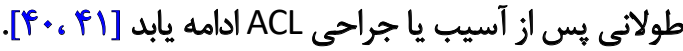

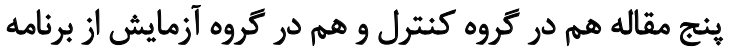

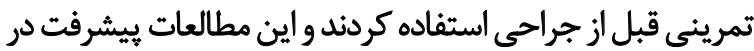

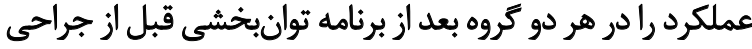

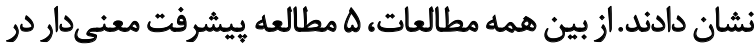

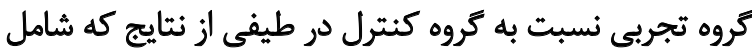

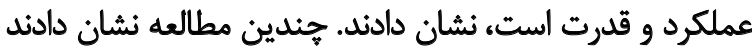

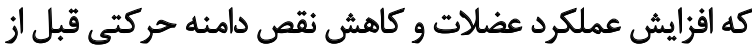

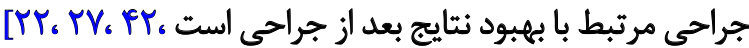

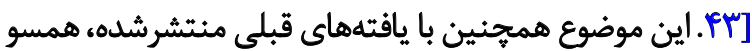

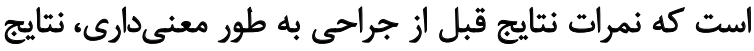

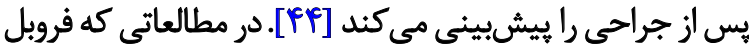

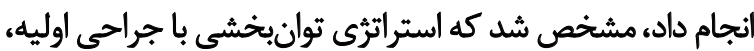

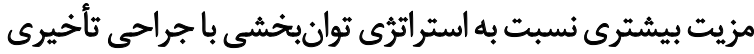

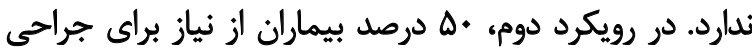

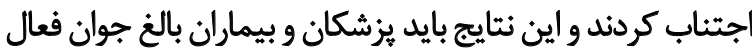

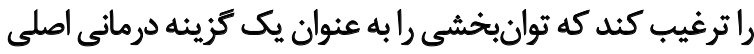

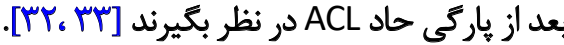

ايتزن و ماكسنيز دريافتند كه يك برنامه ه هفتهاي قبل از

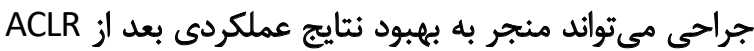

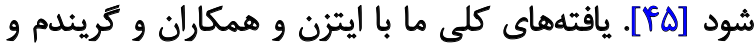

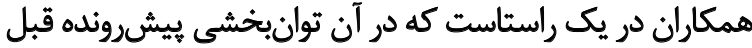

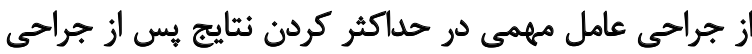

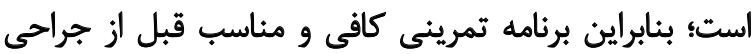

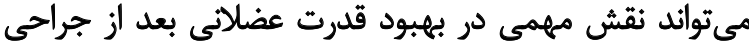

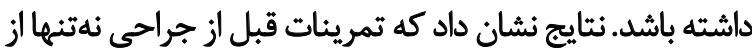

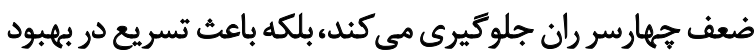

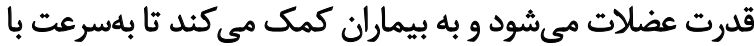

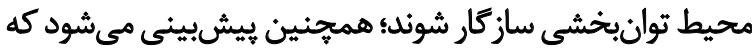

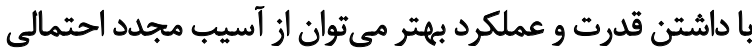

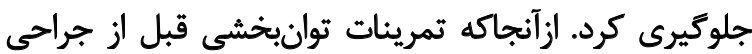

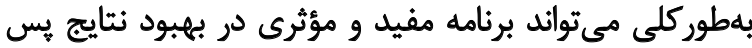

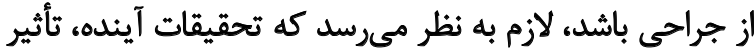

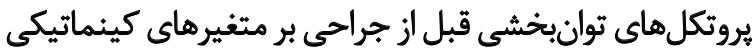
و كينتيكى بس از جراحى را بررسى كنند.

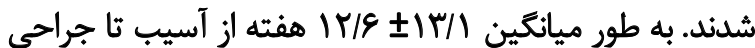

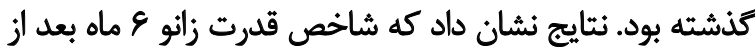

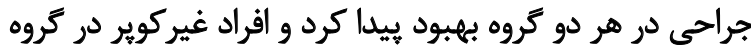

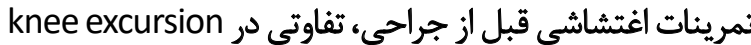

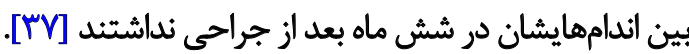

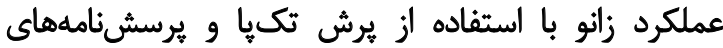

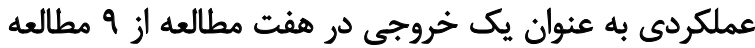

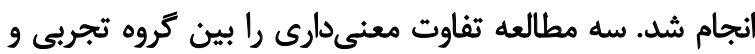

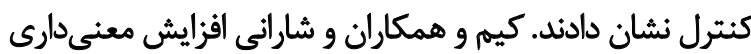

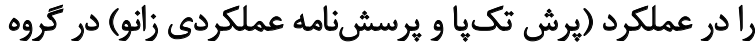

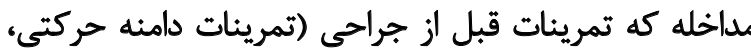

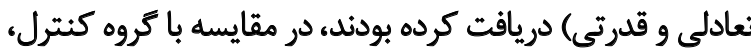

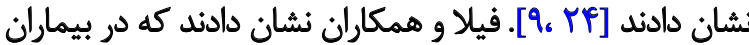

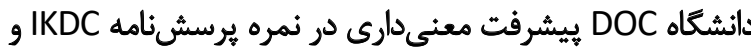

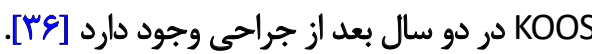

تجهار مطالعه تفاوت معنى دارى رادر عملكرد بين كروهها نشان

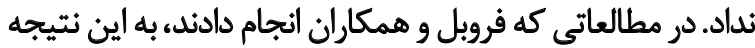

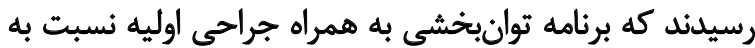

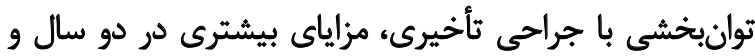

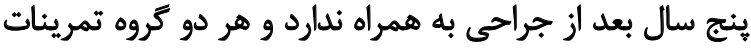

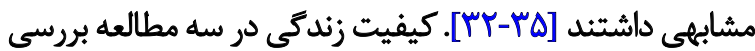

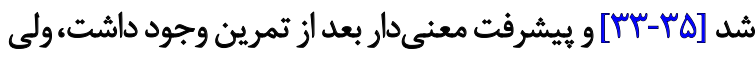

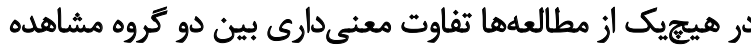

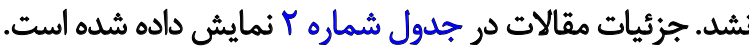

ث

با وجود طيف وسيعى از رويكردهاى قبل از جراحى كه دري اين مقاله مرورى بررسى شدي، نويسندكان اين مقاله

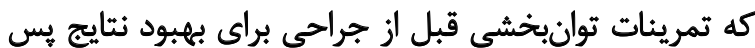

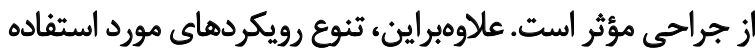

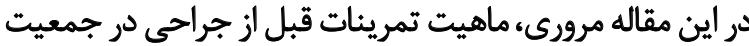

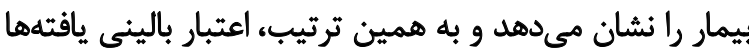

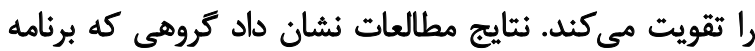

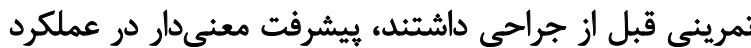

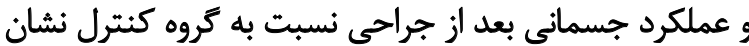

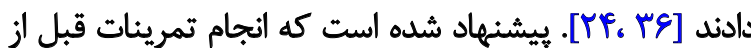

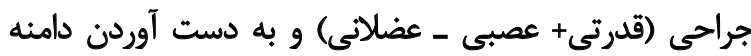

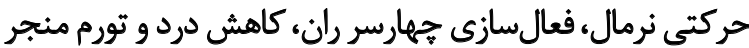

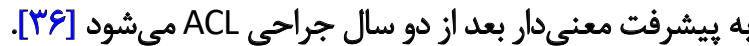

علاوهبراين، با انجام تمرينات قبل از جراحي كه شامل تمرينات

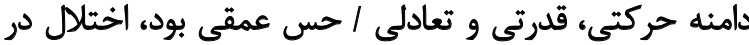

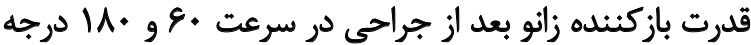

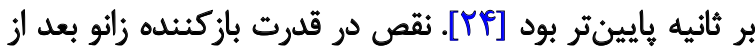




\section{نتيجهَيرى نهايي}

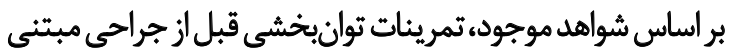

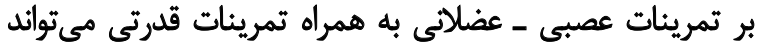

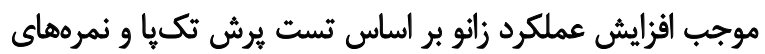

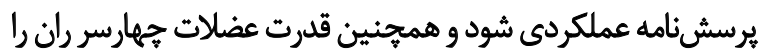

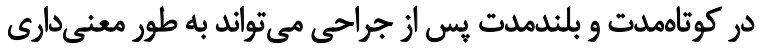

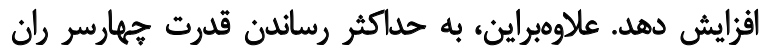

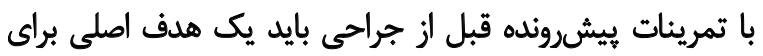
متخصصان به منظور بهبود عملكرد زانو بعد از جراحى باشي باشد.

مالاحظات اخلاقي يبروى از اصول اخلاق يثوهش مقاله از نوع مرورى است به همين دليل نياز به كد اخلاق و ساير اصول اخلاقى ندارد.

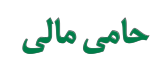

اين مقاله حامى مالى ندارد و برترفته از هيج پاياننامه و يا

$$
\text { طرحى نيست. }
$$

$$
\text { مشاركت ثويسندكّان }
$$

روش رشناسى، تحقيق و بررسى، منابع و نكارش بيشنويس:

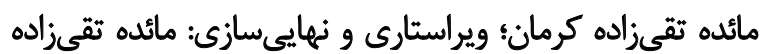

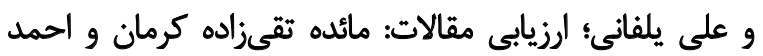

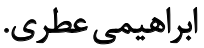

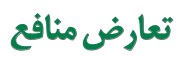

طبق نظر نويسندكان اين مقاله تعارض منافع ندارد. 


\section{References}

[1] Hootman JM, Dick R, Agel J. Epidemiology of collegiate injuries for 15 sports: Summary and recommendations for injury prevention initiatives. J Athl Train. 2007; 42(2):311-9.

[2] Nagano Y, Ida H, Akai M, Fukubayashi T. Gender differences in knee kinematics and muscle activity during single limb drop landing. Knee. 2007; 14(3):218-23. [DOI:10.1016/j.knee.2006.11.008] [PMID]

[3] Kobayashi A, Higuchi H, Terauchi M, Kobayashi F, Kimura M, Takagishi K. Muscle performance after anterior cruciate ligament reconstruction. Int Orthop. 2004; 28(1):48-51. [DOI:10.1007/s00264-0030502-5] [PMID] [PMCID]

[4] Griffin LY, Albohm MJ, Arendt EA, Bahr R, Beynnon BD, DeMaio M, et al. Understanding and preventing noncontact anterior cruciate ligament injuries: A review of the Hunt Valley II meeting, January 2005. Am J Sports Med. 2006; 34(9):1512-32. [DOI:10.1177/0363546506286866] [PMID]

[5] Taghizadeh M, Atri AE, Hashemi Javaheri SAA. The effect of FIFA 11+ injury prevention program on dynamic balance and knee isometric strength of female players in soccer super league. World Family Medicine (WFM) / Middle East Journal of Family Medicine (MEJFM). 2018; 16(7):48-54. [DOI:10.5742/MEWFM.2018.93475]

[6] Zarei M, Abbasi H, Daneshjoo A, Gheitasi M, Johari K, Faude O, et al. The effect of the "11+kids" program on the isokinetic strength of young football players. Int J Sports Physiol Perform. 2019; 15(1):25-30. [DOI:10.1123/ijspp.2018-0827] [PMID]

[7] Atri A, Baharifard R, Khoshraftar N. [Effect of FIFA 11+injury prevention program for eight weeks on the dynamic postural stability of teenage male soccer players in single-leg jump-landing exercises (Persian)]. Saf Promot Inj Prev. 2017; 5(2):79-88.

[8] Soltandoost M, Atri AE, Yazdi NK. [Effect of FIFA 11+Injury prevention program on anterior knee shear force in teenage male soccer players (Persian)]. J Rehab Med. 2017; 6(1):1-9.

[9] Shaarani SR, O'Hare C, Quinn A, Moyna M, Moran R, O'Byrne $J M$. Effect of prehabilitation on the outcome of anterior cruciate ligament reconstruction. Am J Sports Med. 2013; 41(9):21-7. [DOI:10.1177/0363546513493594] [PMID]

[10] Risberg MA, Inger $\mathrm{H}$. The long-term effect of 2 postoperative rehabilitation programs after anterior cruciate ligament reconstruction a randomized controlled clinical trial. Am J Sports Med. 2009; 37(10):1958-66. [DOI:10.1177/0363546509335196] [PMID]

[11] Palmieri-Smith RM, Thomas AC, Wojtys EM. Maximizing quadriceps strength after ACL reconstruction. Clin Sports Med. 2008; 27(3):405-24. [DOI:10.1016/j.csm.2008.02.001] [PMID]

[12] Hurley MV. The role of muscle weakness in the pathogenesis of osteoarthritis. Rheum Dis Clin North Am. 1999; 25(2):283-98. [DOI:10.1016/ S0889-857X(05)70068-5]

[13] Lewek M, Rudolph K, Axe MS-ML. The effect of insufficient quadriceps strength on gait after anterior cruciate ligament reconstruction. Clin Biomech. 2002; 17(1):56-63. [DOI:10.1016/S0268-0033(01)00097-3]

[14] Shelbourne KD F DA. Timing of surgery in acute anterior cruciate ligament tears on the return of quadriceps muscle strength after reconstruction using an autogenous patellar tendon graft. Am J Sports Med. 1995; 23(6):686-9. [DOI:10.1177/036354659502300609] [PMID]

[15] Wasilewski SA, Covall DJ Cohen S. Effect of surgical timing on recovery and associated injuries after anterior cruciate ligament reconstruction. Am J Sports Med. 1993; 21(3):338-42. [DOI:10.1177/03635 4659302100302] [PMID]
[16] Cosgarea AJ, Sebastianelli WJ, DeHaven KE. Prevention of arthrofibrosis after anterior cruciate ligament reconstruction using the central third patellar tendon autograft. Am J Sports Med. 1995; 23(1):87-92. [DOI:10 .1177/036354659502300115] [PMID]

[17] Taghizadeh M. [The relationship between anthropometric characteristics and hip joint flexibility among teenaged girls in football schools (Persian)]. Research on Sports Medicine Technologies. 1391; 2(4):1391-7.

[18] Shelbourne KD, Nitz P. Accelerated rehabilitation after anterior cruciate ligament reconstruction. J Orthop Sports Phys Ther. 1992; 15(6):25664. [DOI:10.2519/jospt.1992.15.6.256] [PMID]

[19] Wilk KE, Andrews JR. Current concepts in the treatment of anterior cruciate ligament disruption. J Orthop Sports Phys Ther. 1992; 15(6):27993. [DOI:10.2519/jospt.1992.15.6.279] [PMID]

[20] Shelbourne KD, Rowdon GA. Anterior cruciate ligament in jury: The competitive athlete. Sports Med. 1994; 17(2):132-40. [DOI:10.2165/00007256-199417020-00005] [PMID]

[21] Keays SL , Bullock-Saxton JE , Newcombe P, Bullock MI. The effectiveness of a pre-operative home-based physiotherapy programme for chronic anterior cruciate ligament deficienc. Physiother Res Int. 2006; 11(4):204-18. [DOI:10.1002/pri.341] [PMID]

[22] Eitzen I, Holm I, Risberg MA. Preoperative quadriceps strength is a significant predictor of knee function two years after anterior cruciate ligament reconstruction. Br J Sports Med. 2009; 43(5):371-6. [DOI:10.1136/ bjsm.2008.057059] [PMID]

[23] Shelbourne KD, Johnson BC. Effects of patellar tendon width and preoperative quadriceps strength on strength return after anterior cruciate ligament reconstruction with ipsilateral bone-patella tendon-bone autograft. Am J Sports Med. 2004; 32(6):1474-8. [DOI:10.1177/0363546503262171] [PMID]

[24] Kim DK, Hwang JH, Park WH. Effects of 4 weeks preoperative exercise on knee extensor strength after anterior cruciate ligament reconstruction. J Phys Ther Sci. 2015; 27(9):2693-6. [DOI:10.1589/ jpts.27.2693] [PMID] [PMCID]

[25] Aggarwal A, Adhya B, Singh Dhillon M. Effectiveness of isokinetic exercises in preoperative anterior cruciate ligament tears rehabilitation. J Postgrad Med, Edu Res. 2016; 50(1):5-8. [DOI:10.5005/jpjournals-10028-1183]

[26] Wilk KE, Arrigo CA. Rehabilitation principles of the anterior cruciate ligament reconstructed knee. Clin Sports Med. 2017; 36(1):189-232. [DOI:10.1016/j.csm.2016.08.012] [PMID]

[27] Logerstedt D, Lynch A, Axe MJ, Snyder-Mackler L. Pre-operative quadriceps strength predicts IKDC2000 scores 6 months after anterior cruciate ligament reconstruction. Knee. 2013; 20(3):208-12. [DOI:10.1016/j. knee.2012.07.011] [PMID] [PMCID]

[28] Grindem H, Granan LP, Risberg MA, Engebretsen L, Snyder-Mackle L, Eitzen I. How does a combined preoperative and postoperative rehabilitation programme influence the outcome of $\mathrm{ACL}$ reconstruction 2 years after surgery? A comparison between patients in the Delaware-Oslo ACL Cohort and the Norwegian National Knee Ligament Registry. Br J Sports Med. 2015; 49(6):385-9. [DOI:10.1136/bjsports-2014-093891] [PMID] [PMCID]

[29] Moher D, Shamseer L, Clarke M, Ghersi D, Liberati A, Petticrew M, et al. Preferred reporting items for systematic review and meta-analysis protocols (PRISMA-P) 2015 statement. Revista Española de Nutrición Humana y Dietética. 2016; 20(2):148-60. 